\title{
STING SNP R293Q Is Associated with a Decreased Risk of Aging-Related Diseases
}

\author{
Lutz Hamann $^{\text {a Juan S. Ruiz-Moreno }}{ }^{\text {b }}$ Malgorzata Szwed ${ }^{c}$ \\ Malgorzata Mossakowska ${ }^{d}$ Linn Lundvall ${ }^{a}$ Ralf R. Schumann ${ }^{a}$

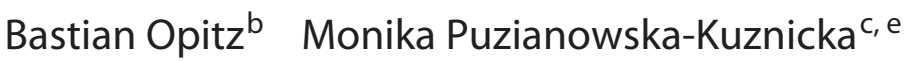

a Department of Microbiology, Infectious Diseases, and Immunology, Charité - University Medical Center, Berlin, Germany; ${ }^{\mathrm{b}}$ Department of Internal Medicine/Infectious Diseases and Pulmonary Medicine, Charité University Medical Center, Berlin, Germany; ${ }^{\circ}$ Department of Human Epigenetics, Mossakowski Medical

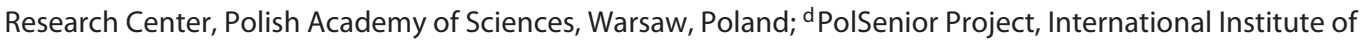

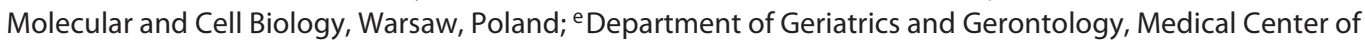
Postgraduate Education, Warsaw, Poland

\section{Keywords}

Senescence · cGAS-STING pathway · Polymorphism

\begin{abstract}
Background: Aging is a multifactorial process driven by several conditions. Among them, inflamm-aging is characterized by chronic low-grade inflammation driving aging-related diseases. The aged immune system is characterized by the senescence-associated secretory phenotype, resulting in the release of proinflammatory cytokines contributing to inflamm-aging. Another possible mechanism resulting in inflamm-aging could be the increased release of dangerassociated molecular patterns (DAMPs) by increased cell death in the elderly, leading to a chronic low-grade inflammatory response. Several pattern recognition receptors of the innate immune system are involved in recognition of DAMPs. The DNA-sensing cGAS-STING pathway plays a pivotal role in combating viral and bacterial infections and rec-
\end{abstract}

\section{KARGER}

(c) 2018 S. Karger AG, Basel

E-Mail karger@karger.com

www.karger.com/ger ognizes DNA released by cell death during the process of aging, which in turn may result in increased inflamm-aging. Objective: The aim of this study was to investigate whether a variation within the STING gene with known impaired function may be associated with protection from aging-related diseases by decreasing the process of inflamm-aging. Methods: STING (Tmem173) R293Q was genotyped in a cohort of 3,397 aged subjects (65-103 years). The distribution of the variant allele in healthy subjects and subjects suffering from aging-associated diseases was compared by logistic regression analysis. Results: We show here that STING 293Q allele carriers were protected from aging-associated diseases ( $O R=0.823, p=0.038$ ). This effect was much stronger in the subgroup of subjects suffering from chronic lung diseases (OR $=0.730, p=0.009)$. Conclusion: Our results indicate that decreased sensitivity of the innate immune receptors is associated with healthy aging, most likely due to a decreased process of inflamm-aging.

(c) 2018 S. Karger AG, Basel
Lutz Hamann, $\mathrm{PhD}$

Department of Microbiology, Infectious Diseases, and Immunology Charité - University Medical Center Berlin

Hindenburgdamm 30, DE-12203 Berlin (Germany)

E-Mail lutz.hamann@charite.de 


\section{Introduction}

The process of aging is defined as a decline in performance/fitness and the onset of aging-related diseases, whereas healthy or successful aging shows a delayed onset of these conditions. Aging is accompanied by the accumulation of senescent cells that has been suggested to be associated with aging-related diseases [1]. The process of senescence affects different systems, e.g., the immune system (immunosenescence) and the proliferative system (replicative or cellular senescence). Immunosenescence describes a profound immune remodulation in the elderly affecting both innate and adaptive immunity. Senescence of the adaptive immune system is mainly characterized by a reduced $\mathrm{T}$-cell receptor repertoire, reduced numbers of naïve T-cells, telomere shortening, and a reduced thymic output resulting in a reduced antigen-specific immune response [2]. Senescence of the innate immune system is characterized by diminished immune responses due to decreased functions of neutrophils, macrophages, and dendritic cells as determined by reduced chemotaxis, phagocytosis, signal transduction, cytokine production upon infections, decreased antigen presentation, and in addition also a change in the natural killer cell subsets [3]. Replicative (cellular) senescence has been described first as a mechanism protecting from tumorigenesis by inducing cell cycle arrest (reviewed by van Deursen [4] and Campisi [1]). Accordingly, classical mechanisms that induce replicative senescence are tumor-associated, such as telomere erosion, DNA lesions, and reactive oxygen species, all known to induce DNA damage response [5]. Senescent cell cycle arrest is achieved by the activation of two tumor suppressor pathways, p53 and pRb [6]. It has been suggested that replicative senescence may not only protect from cancer, but may also drive aging-related diseases, thereby being an example of antagonistic pleiotropy [7]. The most accepted evidence for replicative senescence exhibiting antagonistic pleiotropy regarding aging comes from p53, with high activity being tumorsuppressive but increasing the risk of aging [8]. Possible mechanisms for such an antagonistic pleiotropy are the depletion of tissues from proliferating or stem cell pools and the secretion of factors that disrupt tissue integrity and degenerate extracellular matrix [9]. During the last decade, substantial evidence has been accumulated showing that cellular senescence is indeed also involved in other biological processes such as wound healing, tissue repair, aging, and aging-related diseases (reviewed by van Deursen [4]).
The involvement of cellular senescence in the onset of aging-related diseases has been shown by lifelong removal of senescent cells in mice resulting in a delayed onset of aging-related pathologies. Late-life clearance of senescent cells results in attenuated progression of already established aging-associated diseases [10]. Several senescent mechanisms have been postulated to drive aging-related diseases, e.g., stem cell dysfunction, disruption of extracellular matrix, paracrine senescence, and stimulation of sterile tissue inflammation [4]. The release of proinflammatory cytokines and chemokines, also known as the senescence-associated secretory phenotype (SASP) [11], has been linked to aging-related diseases [12]. The SASP may drive another characteristic of the aged immune system called inflamm-aging $[13,14]$. This describes a process of chronic low-grade inflammation, characterized by elevated baseline levels of proinflammatory mediators such as C-reactive protein (CRP), tumor necrosis factor $\alpha$, interleukin $1 \beta$ (IL- $1 \beta$ ), and IL- 6 in the elderly, which has been shown to be associated with aging-related diseases $[15,16]$.

Inflammatory responses are usually generated following sensing microbial pathogen-associated patterns or endogenous danger-associated molecular patterns (DAMPs) by pattern recognition receptor of the innate immune system [17]. Among others, DNA is an important DAMP recognized by several innate immune receptors, e.g., Toll-like receptor 9, cGAS, IFI16, and AIM2 [18]. Binding of DNA to both IFI16 and cGAS results in activation of STING and a subsequent inflammatory response $[19,20]$. Both DNA receptors are able to sense microbial DNA during infections as well as endogenous DNA, which may be released upon cell death during aging [21]. In particular, mitochondrial DNA release from damaged mitochondria has been shown to increase with age and thus may drive inflamm-aging [22]. At this point, signaling networks for aging or infections may overlap. Cytosolic or cell free DNA originating from the process of aging, carcinogenesis, viral infections, or bacterial infections is a strong inducer of the innate immune response [23].

It has been shown that DNA recognition by IFI16 plays a role in senescence as well as aging-associated diseases via stimulation of STING and subsequent stimulation of interferon $\beta$ expression (reviewed by Choubey and Panchanathan [24]). Furthermore, it has recently been found that innate immune signaling via cGAS also promotes senescence through the secretion of inflammatory cytokines and chemokines and by involving auto- and paracrine signaling. In addition, cGAS as well as STING 


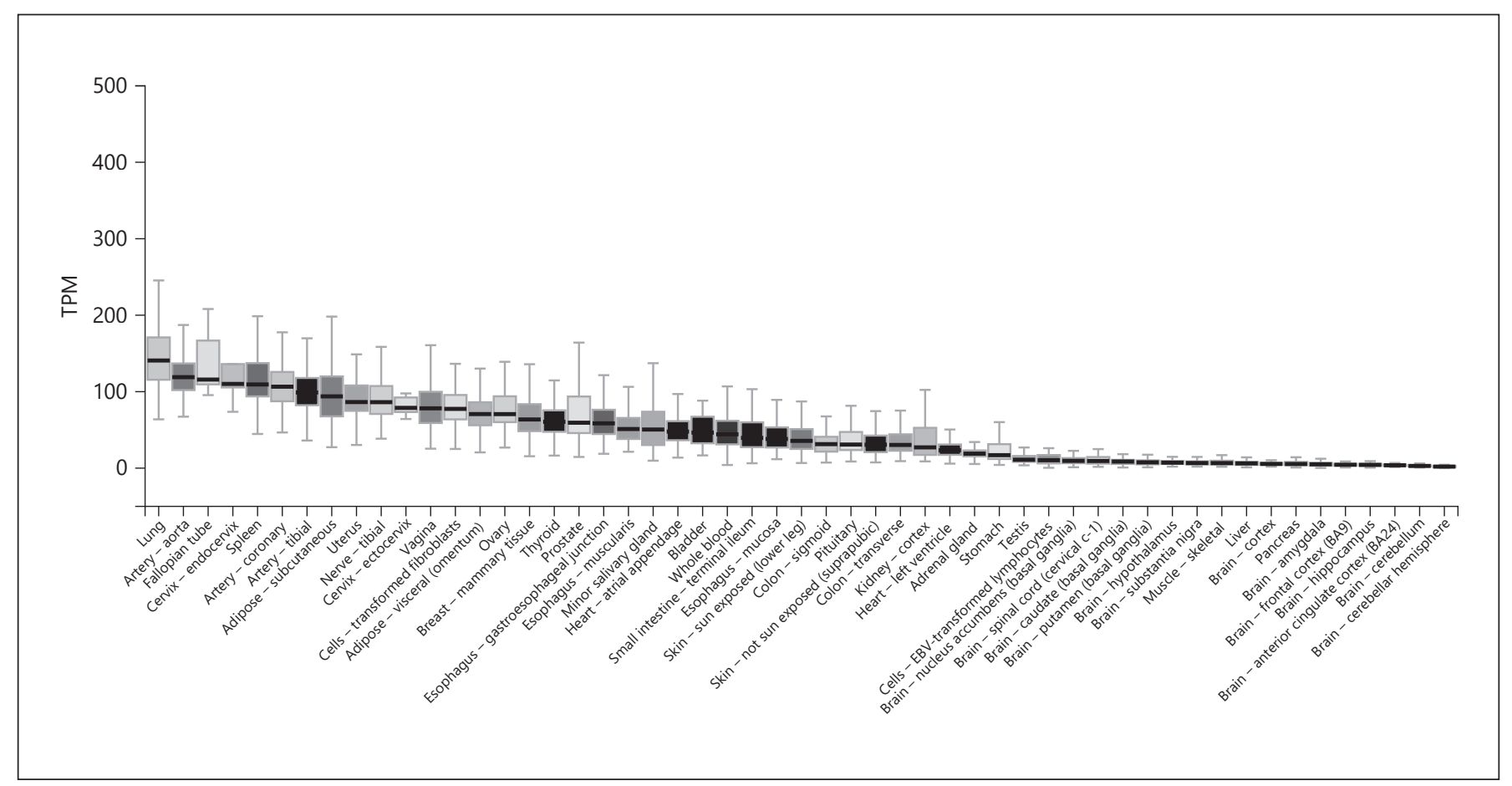

Fig. 1. STING (TMEM173) expression in various tissues from the GTEx Portal.

knockout mice exhibit a reduced SASP upon irradiation compared to wild-type mice $[25,26]$.

A common STING haplotype (HAQ) composed of

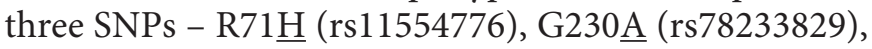
and R293Q (rs7380824) - found in linkage disequilibrium has been described previously by us and others to be hypomorphic [27]. We hypothesize that less signaling by this haplotype may reduce inflamm-aging and therefore be protective against aging-associated diseases, supporting the hypothesis of inflamm-aging being a driving force of human aging by means of antagonistic pleiotropy [28]. We genotyped 3,397 participants of the PolSenior study [29] for STING R293Q (rs7380824) and found allele carriers to be protected from aging-related diseases $(\mathrm{OR}=0.823, p=0.038)$. This effect was much stronger in a small subgroup of current smokers $(\mathrm{OR}=0.391, p=$ $0.001)$.

\section{Subjects and Methods}

\section{Study Cohort}

A total of 3,397 participants of the PolSenior program were analyzed. PolSenior was a multicenter interdisciplinary project designed to assess the health and socioeconomic status of Polish Caucasians aged $\geq 65$ years. The details of the PolSenior recruit- ment are described elsewhere [29]. A detailed questionnaire regarding their medical, social, and economic past and current health status was completed by all project participants. All participants underwent an examination including elements of comprehensive geriatric assessment and donated blood for biochemical and genetic analyses. Steady-state IL-6 levels were measured using ELISA (R\&D Systems, Minneapolis, MN, USA, sensitivity $0.04 \mathrm{pg} / \mathrm{mL}$ ). Subjects with a Mini-Mental State Examination score $<24$, determined according to the Polish version of the MiniMental State Examination test, were classified having cognitive impairment. According to Akbaraly et al. [30] we set IL-6 levels $\leq 1.0 \mathrm{ng} / \mathrm{L}$ as low, $1.1-2.0 \mathrm{ng} / \mathrm{L}$ as intermediate, and $>2.0 \mathrm{ng} / \mathrm{L}$ as high. Low IL-6 levels were used as the reference in logistic regression analysis.

\section{Genotyping}

Genomic DNA was prepared by standard procedures from whole blood. STING SNP R293Q (rs7380824) genotyping was performed by melting curve analysis. Melting curve analysis was carried out employing primers ACC CTG GTA GGC AAT GA (forward), GCT TAG TCT GGT CTT CCT CTT AC (reverse), and FRET-hybridization probes: CCT CAA GTG TCC GGC AGA GTT-FL and LC-red 640-GGC CTG CTC AAG CCT ATC CTC CCG G, resulting in melting points of 67.0 and $59.4^{\circ} \mathrm{C}$ for the wildtype $(\mathrm{C})$ and the mutated $(\mathrm{T})$ allele, respectively.

\section{Statistics}

Binary logistic regression analyses and Mann-Whitney U test were performed employing the IBM SPSS statistics software package version 20.0 (IBM, Munich, Germany). 


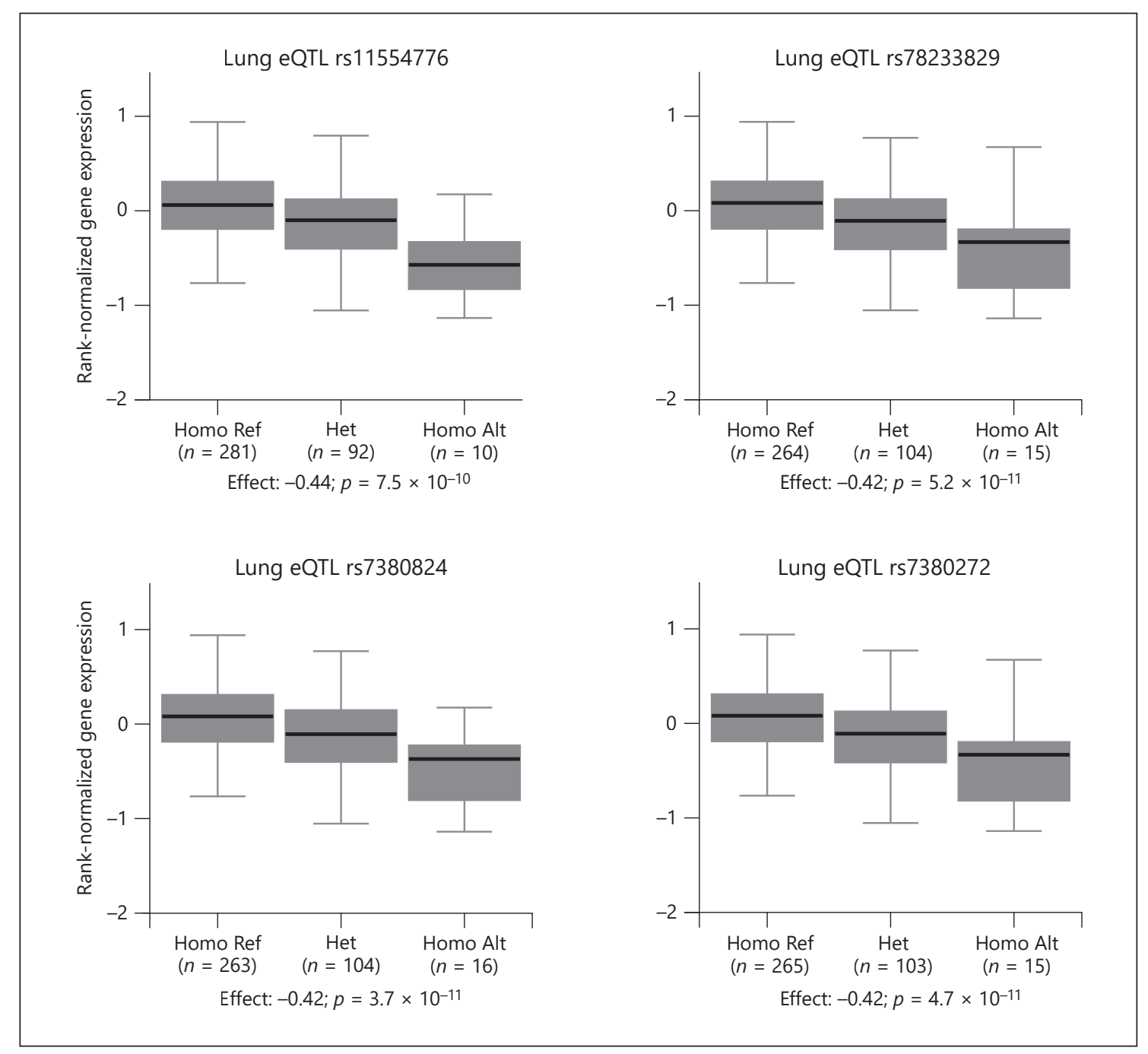

Fig. 2. All HAQ SNPs exhibited lung expression quantitative trait loci according to the GTEx Portal.

\section{Results}

\section{SNP Selection}

Since a functional analysis of certain STING (Tmem173) SNPs, particularly HAQ haplotype SNPs that may affect STING functions, has not been carried out so far, we conducted in silico analyses to choose the most promising SNP in respect to functional consequences in order to detect associations with aging-related diseases. To this end we first checked the GTEx Portal (https://www.gtexportal.org) for STING expression in tissues related to aging-associated diseases, e.g., lung, cardiovascular system, pancreas, and brain. As shown in Figure 1, STING expression was highest in the lung and cardiovascular system and lowest in the brain and pancreas. Since the HAQ haplotype has been associated with decreased protein expression, we next checked the STING gene for cis expression quantitative trait loci employing the GTEx Portal. We found all HAQ SNPs and one additional intron SNP (rs7380272) to be strongly associated with decreased STING expression in lung tissue. All SNPs exhibited similar effect sizes and $p$ values (Fig. 2). These SNPs showed the same association with decreased STING expression in the aorta (data not shown). Next we used the LDlink analysis tool from the National Cancer Institute website (https://ldlink.nci.nih. gov/) based on the 1000 Genomes Project to look for SNPs in linkage disequilibrium within the STING gene 
in the European population that may account for functional effects. Table 1 shows all STING SNPs with $r^{2}>$ 0.90. Three of them (rs6874798, rs7448031, and rs 34703538 ) are located $4 \mathrm{~kb}$ upstream of the coding region and may be part of regulatory elements. However, transcriptional regulation of STING has not been analyzed so far. We next looked up these SNPs within the PolyPhen database at the Ensembl genome browser (www.ensembl.org) and found rs7380824 to be the most promising one to impair STING function. Since mutagenesis experiments of mouse STING also revealed amino acid position 293 to be important for STING function [31], we chose rs7380824 for genotyping.

\section{Common Risk Factors for Aging-Related Diseases in the Study Cohort}

The baseline characteristics of the study cohort are shown in Table 2. The distribution of genotypes was in Hardy-Weinberg equilibrium $(p=0.88)$ and reflected the distribution expected from the 1000 Genomes Project for a European population. We first characterized the study cohort by determining whether common risk factors, such as age, body mass index (BMI; presented as under-/ overweight or obesity), microinflammation (inflammaging, presented as IL-6 steady-state level), and smoking were associated with the phenotype of aging. Therefore, we compared healthy subjects without any aging-related disease $(n=1,068)$ with subjects suffering from one or more aging-related diseases $(n=2,166)$, e.g., cardiovascular disease $(n=844)$, cancer $(n=201)$, chronic lung disease $(n=614)$, type 2 diabetes mellitus (T2DM, $n=$ $673)$, and cognitive impairment $(n=969)$. Univariate analyses of common risk factors (age, BMI, smoking, and microinflammation) showed a significant association with aging-related diseases for all factors, with the exception of smoking. $p$ values for age, BMI (obesity), and intermediate as well as high IL-6 concentrations were < $0.001,0.003,<0.001$, and $<0.001$, respectively. Smoking, past and current combined, showed no significant association $(p=0.507)$ (Table 3$)$. As reported previously, the past and current smoking subjects of our current cohort had a significantly lower age and BMI, which may counteract the risk of smoking [32]. A multivariate analysis of age, BMI, smoking, and microinflammation showed smoking also to be a significant risk factor for the occurrence of aging-related disease. ORs and $p$ values were as follows: $\mathrm{OR}=1.048$ and $p<0.001$ (age), $\mathrm{OR}=1.655$ and $p<0.001$ (BMI: obesity), $\mathrm{OR}=1.205$ and $p=0.022$ (smoking), $\mathrm{OR}=1.478$ and $p=0.005$ (microinflammation: intermediate), and $\mathrm{OR}=1.722$ and $p<0.001$ (microinflam-

Table 1. LDlink analysis showing all SNPs in strong linkage disequilibrium $\left(r^{2}>0.90\right)$ with $r 57380824$

\begin{tabular}{lll}
\hline SNP & $r^{2}$ & Location \\
\hline rs6874798 & 0.94 & $5^{\prime} \mathrm{UTR}$ \\
rs7448031 & 0.94 & $5^{\prime} \mathrm{UTR}$ \\
rs34703538 & 0.94 & $5^{\prime} \mathrm{UTR}$ \\
rs78233829 & 1.0 & G230A \\
rs11554776 & 1.0 & R71H \\
rs7380824 & - & R293Q \\
rs7380272 & 1.0 & intron \\
rs73257329 & 1.0 & intron \\
rs75746446 & 0.94 & $3^{\prime} \mathrm{UTR}$ \\
\hline
\end{tabular}

Table 2. Baseline characteristic of the study subjects in the PolSenior group

Number of subjects

Age range, years

Mean age, years

Males/females

Mean BMI

Smoking: yes/no

Baseline IL-6, ng/L

Disease-free

CVD

Cancer

Chronic lung disease

T2DM

Cognitive impairment

STING

RR

RQ

QQ

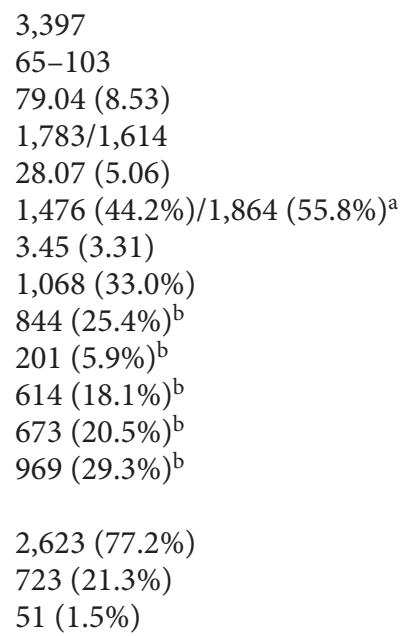

Figures in parentheses are SD or \%. BMI, body mass index; CVD, cardiovascular disease; IL, interleukin; T2DM, type 2 diabetes mellitus. ${ }^{a}$ No data for some subjects. ${ }^{b}$ Some subjects had more than one disease.

mation: high) (Table 4). These data show that common risk factors such as age, BMI, smoking, and microinflammation were strongly associated with aging-associated diseases in our cohort.

\section{STING Allele 293Q Is Associated with Protection from} Aging-Associated Diseases

To analyze whether the nonfunctional STING allele 293Q is associated with aging-related diseases, we analyzed the whole cohort by multivariate logistic regression analysis, comparing healthy subjects with those 
Table 3. Association of common risk factors with combined aging-related diseases: univariate analysis

\begin{tabular}{|c|c|c|c|c|}
\hline Risk factor & $p$ value & Healthy & Disease & OR (95\% CI) \\
\hline Age & $<0.001$ & $76.8(8.1)$ & $79.9(8.4)$ & \\
\hline \multicolumn{5}{|l|}{ BMI } \\
\hline Under-/overweight & 0.983 & & & $0.998(0.831-1.119)$ \\
\hline Obesity & 0.003 & & & $1.358(1.112-1.659)$ \\
\hline \multicolumn{5}{|l|}{ IL-6 } \\
\hline Intermediate & $<0.001$ & & & $1.673(1.285-2.180)$ \\
\hline High & $<0.001$ & & & $2.407(1.871-3.096)$ \\
\hline Smoking & 0.507 & & & $1.052(0.906-1.220)$ \\
\hline
\end{tabular}

Figures are presented as mean (SD) unless indicated otherwise. Age was analyzed by Mann-Whitney U test. BMI, microinflammation (IL-6), and smoking were analyzed by logistic regression with BMI 18.5-24.9, IL-6 $\leq 1$ $\mathrm{ng} / \mathrm{L}$, and no smoking as references. Predictors were as follows: BMI $<18.5$ or 25-29.9 (under-/overweight) and BMI >30 (obesity), IL-6 1.1-2.0 ng/L (intermediate) and >2 ng/L (high), and smoking. BMI, body mass index; IL, interleukin.

Table 4. Association of common risk factors with combined agingrelated diseases: multivariate analysis

\begin{tabular}{lrl}
\hline Risk factor & $p$ value & OR (95\% CI) \\
\hline Age & $<0.001$ & $1.048(1.037-1.059)$ \\
BMI $\quad$ & \\
$\quad$ Under-/overweight & 0.381 & $1.091(0.898-1.326)$ \\
$\quad$ Obesity & $<0.001$ & $1.655(1.330-2.059)$ \\
Smoking & 0.022 & $1.205(1.026-1.415)$ \\
IL-6 & & \\
$\quad$ Intermediate & 0.005 & $1.478(1.126-1.939)$ \\
$\quad$ High & $<0.001$ & $1.722(1.323-2.243)$ \\
\hline
\end{tabular}

Analysis was carried out by logistic regression with BMI 18.524.9, IL- $6 \leq 1 \mathrm{ng} / \mathrm{L}$, and no smoking as references. Predictors were as follows: BMI $<18.5$ or $25-29.9$ (under-/overweight) and BMI $>30$ (obesity), IL-6 1.1-2.0 ng/L (intermediate) and > 2 ng/L (high), and past and current smoking. BMI, body mass index; IL, interleukin.

suffering from one or more aging-related diseases. Common risk factors such as age, BMI, microinflammation, and smoking were added as cofactors. As shown in Table 5, STING allele 293Q carriage significantly protected from aging-related diseases $(\mathrm{OR}=0.823,95 \%$ CI $0.683-0.89, p=0.038$ ). The frequency of STING 293Q carriers was $22.8 \%$, with $21.3 \%$ being heterozygous and $1.5 \%$ homozygous. Analysis of separate heterozygous and homozygous genotypes showed a significant association of heterozygous genotypes $(\mathrm{OR}=$ $0.820,95 \%$ CI $0.678-0.992, p=0.041)$, whereas homo-
Table 5. Multivariate analysis of common risk factors and STING 293Q allele carriers for combined aging-related diseases

\begin{tabular}{lrl}
\hline Risk factor & $p$ value & OR $(95 \% \mathrm{CI})$ \\
\hline Age & $<0.001$ & $1.048(1.037-1.059)$ \\
BMI & & \\
$\quad$ Under-/overweight & 0.317 & $1.105(0.908-1.344)$ \\
$\quad$ Obesity & $<0.001$ & $1.686(1.354-2.100$ \\
Smoking & 0.029 & $1.197(1.019-1.406)$ \\
IL-6 & & \\
$\quad$ Intermediate & 0.008 & $1.444(1.098-1.897)$ \\
$\quad$ High & $<0.001$ & $1.689(1.295-2.203)$ \\
STING & & \\
$\quad$ RQ/QQ & 0.038 & $0.822(0.683-0.989)$ \\
$\quad$ RQ & 0.041 & $0.820(0.678-0.992)$ \\
$\quad$ QQ & 0.633 & $0.852(0.441-1.645)$ \\
\hline
\end{tabular}

Analysis was done by logistic regression with BMI 18.5-24.9, IL- $6 \leq 1 \mathrm{ng} / \mathrm{L}$, no smoking, and STING R/R as references. Predictors were as follows: BMI $<18.5$ or $25-29.9$ (under-/overweight) and BMI $>30$ (obesity), IL-6 1.1-2.0 ng/L (intermediate) and >2 ng/L (high), smoking (past and current), as well as combined or separate R/Q and Q/Q genotypes. BMI, body mass index; IL, interleukin.

zygous genotypes showed no significant association, most likely due to the low sample number. Therefore, only combined heterozygous and homozygous genotypes were analyzed subsequently. Common risk factors showed nearly the same significance levels as shown before without addition of STING R293Q as a risk factor (Table 4). 
Table 6. Multivariate analysis of common risk factors and STING 293Q allele carriers for certain aging-related diseases

\begin{tabular}{|c|c|c|}
\hline Risk factor & $p$ value & OR (95\% CI) \\
\hline \multicolumn{3}{|c|}{ Cardiovascular disease $(n=734)$} \\
\hline Age & 0.329 & $1.005(0.995-1.016)$ \\
\hline \multicolumn{3}{|r|}{ 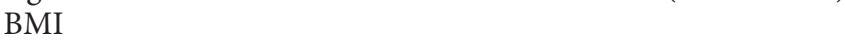 } \\
\hline Under-/overweight & 0.014 & $1.313(1.056-1.634)$ \\
\hline Obesity & 0.002 & $1.465(1.157-1.854)$ \\
\hline \multicolumn{3}{|l|}{ IL-6 } \\
\hline Intermediate & 0.229 & $1.225(0.880-1.705)$ \\
\hline High & 0.018 & $1.469(1.068-2.021)$ \\
\hline Smoking & $<0.001$ & $1.444(1.217-1.712)$ \\
\hline \multicolumn{3}{|l|}{ STING } \\
\hline RQ/QQ & 0.925 & $0.990(0.810-1.211)$ \\
\hline \multicolumn{3}{|c|}{ Chronic lung disease $(n=546)$} \\
\hline Age & 0.006 & $1.017(1.005-1.029)$ \\
\hline \multicolumn{3}{|l|}{ BMI } \\
\hline Under-/overweight & 0.257 & $1.148(0.904-1.457)$ \\
\hline Obesity & 0.061 & $1.282(0.989-1.662)$ \\
\hline \multicolumn{3}{|l|}{ IL-6 } \\
\hline Intermediate & 0.281 & $1.222(0.844-1.770)$ \\
\hline High & 0.090 & $1.362(0.953-1.946)$ \\
\hline Smoking & $<0.001$ & $1.652(1.366-1.998)$ \\
\hline \multicolumn{3}{|l|}{ STING } \\
\hline RQ/QQ & 0.009 & $0.730(0.576-0.924)$ \\
\hline \multicolumn{3}{|l|}{ Cancer $(n=183)$} \\
\hline Age & 0.657 & $1.004(0.985-1.024)$ \\
\hline \multicolumn{3}{|l|}{ BMI } \\
\hline Under-/overweight & 0.069 & $0.718(0.503-1.026)$ \\
\hline Obesity & 0.074 & $0.693(0.464-1.036)$ \\
\hline \multicolumn{3}{|l|}{ IL-6 } \\
\hline Intermediate & 0.969 & $0.989(0.577-1.697)$ \\
\hline High & 0.866 & $0.956(0.567-1.611)$ \\
\hline Smoking & 0.008 & $1.511(1.113-2.051)$ \\
\hline \multicolumn{3}{|l|}{ STING } \\
\hline RQ/QQ & 0.137 & $0.745(0.506-1.098)$ \\
\hline
\end{tabular}

\begin{tabular}{lrr}
\hline Risk factor & $p$ value & OR $(95 \% \mathrm{CI})$ \\
\hline T2DM $(n=597)$ & & \\
Age & 0.786 & $1.002(0.990-1.014)$ \\
BMI & & \\
$\quad$ Under-/overweight & $<0.001$ & $1.801(1.373-2.363)$ \\
$\quad$ Obesity & $<0.001$ & $3.667(2.780-4.837)$ \\
IL-6 & & \\
$\quad$ Intermediate & 0.995 & $0.999(0.709-1.408)$ \\
$\quad$ High & 0.884 & $1.025(0.735-1.429)$ \\
Smoking & 0.482 & $0.935(0.775-1.128)$ \\
STING & & \\
$\quad$ RQ/QQ & 0.902 & $0.986(0.793-1.227)$ \\
\hline Cognitive impairment $(n=833)$ & & \\
Age & $<0.001$ & $1.095(1.083-1.108)$ \\
BMI & & \\
$\quad$ Under-/overweight & 0.021 & $0.780(0.631-0.963)$ \\
$\quad$ Obesity & 0.238 & $0.868(0.687-1.098)$ \\
IL-6 & & \\
$\quad$ Intermediate & 0.058 & $1.428(0.987-2.066)$ \\
$\quad$ High & $<0.001$ & $1.898(1.330-2.708)$ \\
Smoking & 0.001 & $0.735(0.616-0.878)$ \\
STING & 0.645 & $0.953(0.775-1.171)$ \\
$\quad$ RQ/QQ & & \\
\hline
\end{tabular}

Analyses were carried out by logistic regression with BMI 18.524.9, IL-6 $\leq 1 \mathrm{ng} / \mathrm{L}$, no smoking, and STING R/R as references. Predictors were as follows: BMI $<18.5$ or $25-29.9$ (under-/ overweight) and BMI $>30$ (obesity), IL-61.1-2.0ng/L(intermediate) and $>2 \mathrm{ng} / \mathrm{L}$ (high), past and current smoking, as well as combined $\mathrm{R} / \mathrm{Q}$ and $\mathrm{Q} / \mathrm{Q}$ genotypes. BMI, body mass index; IL, interleukin; T2DM, type 2 diabetes mellitus.

STING 293Q Is Strongly Associated with Protection from Chronic Lung Diseases

We next analyzed whether this protective effect is also true for the certain aging-associated diseases. We again performed a multivariate regression analysis for all common risk factors and STING 293Q comparing subjects suffering from cardiovascular diseases, chronic lung diseases, cancer, T2DM, or cognitive impairment with subjects that do not. As shown in Table 6, a significant protection by STING 293Q was only found for chronic lung diseases $(\mathrm{OR}=0.730,95 \%$ CI $0.576-0.924, p=0.009)$. Other significant risk factors were age $(\mathrm{OR}=1.017,95 \%$ CI 1.005-1.029, $p=0.006)$ and smoking $(\mathrm{OR}=1.652$, $95 \%$ CI 1.366-1.998, $p<0.001)$. As expected, smoking exhibited the strongest association with chronic lung dis- eases. Significant risk factors for cardiovascular diseases were again smoking $(\mathrm{OR}=1.444,95 \%$ CI $1.217-1.712$, $p<0.001)$, high microinflammation $(\mathrm{OR}=1.469,95 \% \mathrm{CI}$ $1.068-2.021, p=0.018)$, and obesity $(\mathrm{OR}=1.465,95 \% \mathrm{CI}$ $1.157-1.854, p=0.002)$. For cancer we found only smoking to be a significant risk factor $(\mathrm{OR}=1.511,95 \% \mathrm{CI}$ $1.113-2.051, p=0.008)$. As expected, BMI showed a strong association with T2DM (under/overweight: $\mathrm{OR}=$ $1.801,95 \%$ CI $1.373-2.363, p<0.001$; obesity: OR = $3.667,95 \%$ CI $2.780-4.837, p<0.001)$. Cognitive impairment, known to be strongly associated with age and inflammation, displayed a strong association with age $(\mathrm{OR}=1.095,95 \%$ CI 1.083-1.108, $p<0.001)$, high microinflammation $(\mathrm{OR}=1.898,95 \%$ CI $1.330-2.708, p<$ $0.001)$, and smoking $(\mathrm{OR}=0.735,95 \%$ CI $0.616-0.878$, 
Table 7. STING 293 Q allele carriers with chronic lung disease exhibit decreased baseline levels of inflammatory markers

\begin{tabular}{lllll}
\hline Genotype & CRP, mg/L & $p$ value & IL-6, ng/L & $p$ value \\
\hline RR & $5.95(8.50)$ & & $3.95(3.48)$ & \\
RQ/QQ & $4.85(7.30)$ & 0.037 & $3.65(3.30)$ & 0.190 \\
\hline
\end{tabular}

Figures are presented as mean (SD). $p$ values were determined by Mann-Whitney U test. CRP, C-reactive protein; IL, interleukin.

$p<0.001)$. The protective effect of smoking may be due to the previously mentioned younger age of smokers in our cohort.

\section{STING 293Q Results in Decreased Inflammatory \\ Markers in Subjects Suffering from Chronic Lung \\ Diseases}

According to our hypothesis, we next analyzed whether STING 293Q allele carriers showed decreased steadystate levels of inflammatory markers CRP and IL-6 in subjects suffering from chronic lung diseases. Allele carriers $(n=114)$ showed lower baseline CRP levels than wild-type subjects $(n=483)$ (mean $4.85 \mathrm{mg} / \mathrm{L}, \mathrm{SD}=7.30$, and mean $5.95 \mathrm{mg} / \mathrm{L}, \mathrm{SD}=8.50$, respectively, $p=0.037$ ), as determined by Mann-Whitney $\mathrm{U}$ test (Table 7). Regarding IL-6, allele carriers $(n=113)$ showed lower baseline IL-6 levels than wild-type subjects $(n=473)$ (mean $3.65 \mathrm{ng} / \mathrm{L}, \mathrm{SD}=3.30$, and mean $3.95 \mathrm{ng} / \mathrm{L}, \mathrm{SD}=3.48$, respectively); however, this difference failed to reach statistical significance $(p=0.190)$.

\section{STING 293Q Effects Are Much More Pronounced in Current Smokers}

Since it is well known that cigarette smoke induces oxidative stress, DNA damage, and necrosis resulting in increased release of DNA that in turn may trigger the STING pathway, we analyzed a subgroup of 320 current smokers of our cohort for STING 293Q effects on agingrelated diseases. Since this subgroup was relatively small, we performed a univariate regression analysis and did not include the common risk factors as before. Table 8 shows a significant protective effect of STING 293Q regarding combined aging-related diseases $(\mathrm{OR}=0.391, p=0.001)$. A subgroup analysis for certain aging-related diseases revealed a significant protective effect for cognitive impairment $(\mathrm{OR}=0.439, p=0.042)$. Regarding cardiovascular diseases and T2DM, effects were of borderline significance $(\mathrm{OR}=0.507, p=0.096$, and $\mathrm{OR}=0.415, p=0.055$,
Table 8. Univariate analysis of STING 293Q allele carriers for certain aging-related diseases among current smokers

\begin{tabular}{lll}
\hline Disease & $p$ value & OR $(95 \%$ CI $)$ \\
\hline Combined diseases $(n=182)$ & 0.001 & $0.391(0.222-0.686)$ \\
Cardiovascular disease $(n=62)$ & 0.096 & $0.507(0.228-1.127)$ \\
Chronic lung disease $(n=63)$ & 0.288 & $0.670(0.321-1.402)$ \\
Cancer $(n=20)$ & 0.107 & $0.189(0.025-1.436)$ \\
T2DM $(n=53)$ & 0.055 & $0.415(0.169-1.020)$ \\
Cognitive impairment $(n=69)$ & 0.042 & $0.439(0.199-0.972)$ \\
\hline
\end{tabular}

Analysis was done by logistic regression with STING R/R as reference and combined $\mathrm{R} / \mathrm{Q}$ and $\mathrm{Q} / \mathrm{Q}$ genotypes as predictor. T2DM, type 2 diabetes mellitus.

respectively). STING 293Q failed to show any effect on chronic lung disease in this subgroup, most likely due to the overwhelming effect of current smoking.

\section{Discussion}

In order to analyze the impact of STING R293Q on aging-associated diseases, we genotyped 3,397 samples of the PolSenior group and correlated the genotypes with the occurrence of aging-related diseases such as cardiovascular diseases, chronic lung diseases, cancer, T2DM, and cognitive impairment. We found an overall protective effect of the STING 293Q allele regarding aging-associated diseases $(\mathrm{OR}=0.823, p=0.038)$. This effect relies on a strong association with chronic lung diseases $(\mathrm{OR}=$ $0.730, p=0.009$ ). All other aging-related diseases failed to show any significant association with STING 293Q. Common risk factors showed the expected disease associations, smoking + microinflammation + BMI (cardiovascular diseases), age + smoking (chronic lung diseases), smoking (cancer), BMI (T2DM), and age + microinflammation (cognitive impairment) being strong risk factors. STING 293Q allele carriers suffering from chronic lung diseases revealed lower baseline levels of CRP $(p=0.037)$. IL-6 baseline levels were also lower, but not significantly. The protective effects of STING 293Q were much stronger in the subgroup of current smokers (OR $=0.391, p=$ 0.001 ) for combined aging-related diseases. Furthermore, in this subgroup, STING 293Q was significantly protective regarding cognitive impairment $(\mathrm{OR}=0.439, p=$ 0.042 ) and showed a trend for being protective regarding $\mathrm{T} 2 \mathrm{DM}$ and cardiovascular disease $(\mathrm{OR}=0.415, p=0.055$, and $\mathrm{OR}=0.507, p=0.096$, respectively). 
The strong influence of STING 293Q on chronic lung diseases could potentially be explained as follows: The increase in life expectancy during the last decades results in an increase of morbidity and mortality attributed to chronic lung disease, whereas the incidence of other aging-related diseases remains stable or decreased [33], indicating that particularly lung diseases are affected by aging. Furthermore, immunosenescence, particularly inflamm-aging, was suggested to predispose the elderly for pulmonary infection and to exacerbate respiratory diseases including asthma, COPD, and pulmonary fibrosis (reviewed by Murray and Chotirmall [34]). In addition to inflamm-aging, potentially driven by the cGAS-STING pathway, the finding that viral infections are known to be the major cause of exacerbations of COPD and asthma [35] could additionally be a reason for the strong association of STING 293Q with chronic lung diseases. Viral infections known to stimulate innate immune response via the cGAS-STING pathway may lead to an overwhelming immune response in chronic lung diseases in the elderly. Accordingly, we found decreased inflammatory markers in STING 293Q carriers in the subgroup of subjects suffering from chronic lung diseases.

The finding that STING 293Q effects are stronger and affect a broader spectrum of aging-associated diseases in the subgroup of current smokers is of special interest, although this subgroup is relative small and results should be interpreted carefully. Smoking could be seen as an "aging accelerator" and genetics of aging may be better visible in this group. Cigarette smoke is known to be a strong risk factor for aging-associated diseases such as cancer, cardiovascular diseases, T2DM, and chronic lung diseases [36]. Telomere shortening, one pivotal feature of aging, is accelerated in smokers [37], and in addition, inflammatory markers such as CRP and tumor necrosis factor a were found to be elevated in smokers, potentially driving the process of inflamm-aging [38]. Within the small subgroup of 320 current smokers, the protective effect of STING 293Q regarding combined aging-related diseases was stronger compared to the whole study group and - of note - of borderline significance for all aging-related diseases, with the exception of chronic lung diseases and cancer. For chronic lung diseases, smoking is by far the most relevant risk factor, which may explain STING 293Q failing to show any association within this subgroup. We could not find an association either with cancer in current smokers; however, this subgroup was the smallest one, resulting in no significant association although the OR of 0.107 was consistent with protection.

STING SNP R293Q and Aging-Related Diseases
Replicative senescence is the most accepted example for antagonistic pleiotropy [39]. Here, we present first evidence that one aspect of immunosenescence, i.e., the process of inflamm-aging, may also be an example for antagonistic pleiotropy. The mechanism of antagonistic pleiotropy can simply be seen as investment in early life, resulting in increased reproduction but having negative effects in later life. A highly sensitive immune system is per se of advantage; however, in later life when several aging mechanisms may result in increased cell death due to telomere shortening, oxidative stress or accumulation of mutations resulting in increased release of DAMPs, it may drive the process of inflamm-aging. Our data fit well recent studies showing innate immune sensing via the cGAS-STING pathway to drive senescence by regulating SASP factors $[25,26]$. However, aging is a multifactorial process, as reviewed by López-Otín et al. [40]. Inflammaging is only one among several aspects of aging and, furthermore, cGAS-STING is only one out of several pathways triggering the innate immune response. Therefore, larger studies analyzing functional SNPs within all pathways regulating inflamm-aging must be carried out to confirm this hypothesis.

Future medicine may concentrate on personalized medicine, including SNP screening, potentially predicting the need of an individual for closer medical checkups in order to reduce the frequency of aging-related diseases. This individualized care of the elderly patient may reduce mortality and overall health care cost.

\section{Statement of Ethics}

All participants gave written informed consent for participation in the study. All investigations were carried out in accordance with the ethical guidelines of the 1975 Declaration of Helsinki. The study was approved by the Bioethics Commission of the Medical University of Silesia in Katowice.

\section{Disclosure Statement}

The authors have no conflicts of interest to declare.

\section{Funding Sources}

This work was supported by Charité - University Medical Center Berlin (grant 2007-486), the Berliner Krebsgesellschaft e.V. (all to R.R. Schumann), and by the Polish Ministry of Science and Higher Education (grant PBZ-MEiN-9/2/2006 - K143/P01/2007/1 to M. Puzianowska-Kuznicka, M. Szwed, and M. Mossakowska).

Gerontology 2019;65:145-154 


\section{References}

1 Campisi J. Senescent cells, tumor suppression, and organismal aging: good citizens, bad neighbors. Cell. 2005 Feb;120(4):513-22.

2 Vallejo AN. Immune remodeling: lessons from repertoire alterations during chronological aging and in immune-mediated disease. Trends Mol Med. 2007 Mar;13(3):94-102.

3 Solana R, Tarazona R, Gayoso I, Lesur O, Dupuis G, Fulop T. Innate immunosenescence: effect of aging on cells and receptors of the innate immune system in humans. Semin Immunol. 2012 Oct;24(5):331-41.

4 van Deursen JM. The role of senescent cells in ageing. Nature. 2014 May;509(7501):439-46.

5 Sedelnikova OA, Horikawa I, Zimonjic DB, Popescu NC, Bonner WM, Barrett JC. Senescing human cells and ageing mice accumulate DNA lesions with unrepairable double-strand breaks. Nat Cell Biol. 2004 Feb;6(2):168-70.

6 Bringold F, Serrano M. Tumor suppressors and oncogenes in cellular senescence. Exp Gerontol. 2000 May;35(3):317-29.

7 Hayflick L, Moorhead PS. The serial cultivation of human diploid cell strains. Exp Cell Res. 1961 Dec;25(3):585-621.

8 Ungewitter E, Scrable H. Antagonistic pleiotropy and p53. Mech Ageing Dev. 2009 JanFeb;130(1-2):10-7

9 Campisi J. Cancer and ageing: rival demons? Nat Rev Cancer. 2003 May;3(5):339-49.

10 Baker DJ, Wijshake T, Tchkonia T, LeBrasseur NK, Childs BG, van de Sluis B, et al. Clearance of p16Ink4a-positive senescent cells delays ageing-associated disorders. Nature. 2011 Nov;479(7372):232-6.

11 Rodier F, Coppé JP, Patil CK, Hoeijmakers WA, Muñoz DP, Raza SR, et al. Persistent DNA damage signalling triggers senescenceassociated inflammatory cytokine secretion. Nat Cell Biol. 2009 Aug;11(8):973-9.

12 Byun HO, Lee YK, Kim JM, Yoon G. From cell senescence to age-related diseases: differential mechanisms of action of senescenceassociated secretory phenotypes. BMB Rep. 2015 Oct;48(10):549-58.

13 Franceschi C, Bonafè M, Valensin S, Olivieri F, De Luca M, Ottaviani E, et al. Inflamm-aging. An evolutionary perspective on immunosenescence. Ann N Y Acad Sci. 2000 Jun; 908(1):244-54.

14 Franceschi C, Capri M, Monti D, Giunta S, Olivieri F, Sevini F, et al. Inflammaging and anti-inflammaging: a systemic perspective on aging and longevity emerged from studies in humans. Mech Ageing Dev. 2007 Jan;128(1): 92-105.
15 Franceschi C, Campisi J. Chronic inflammation (inflammaging) and its potential contribution to age-associated diseases. J Gerontol A Biol Sci Med Sci. 2014 Jun;69 Suppl 1:S4-9.

16 Puzianowska-Kuźnicka M, Owczarz M, Wieczorowska-Tobis K, Nadrowski P, Chudek J, Slusarczyk P, et al. Interleukin-6 and C-reactive protein, successful aging, and mortality: the PolSenior study. Immun Ageing. 2016 Jun; 13:21.

17 Takeuchi O, Akira S. Pattern recognition receptors and inflammation. Cell. 2010 Mar; 140(6):805-20.

18 Paludan SR. Activation and regulation of DNA-driven immune responses. Microbiol Mol Biol Rev. 2015 Jun;79(2):225-41.

19 Ablasser A, Goldeck M, Cavlar T, Deimling T, Witte G, Röhl I, et al. cGAS produces a $2^{\prime}-5^{\prime}$-linked cyclic dinucleotide second messenger that activates STING. Nature. 2013 Jun;498(7454):380-4.

20 Unterholzner L, Keating SE, Baran M, Horan KA, Jensen SB, Sharma S, et al. IFI16 is an innate immune sensor for intracellular DNA. Nat Immunol. 2010 Nov;11(11):997-1004.

21 Freitas AA, de Magalhães JP. A review and ap praisal of the DNA damage theory of ageing. Mutat Res. 2011 Jul-Oct;728(1-2):12-22.

22 Pinti M, Cevenini E, Nasi M, De Biasi S, Salvioli S, Monti D, et al. Circulating mitochondrial DNA increases with age and is a familiar trait: implications for "inflamm-aging”. Eur J Immunol. 2014 May;44(5):1552-62.

23 Keating SE, Baran M, Bowie AG. Cytosolic DNA sensors regulating type I interferon induction. Trends Immunol. 2011 Dec;32(12): 574-81.

24 Choubey D, Panchanathan R. IFI16, an amplifier of DNA-damage response: role in cellular senescence and aging-associated inflammatory diseases. Ageing Res Rev. 2016 Jul;28: 27-36.

25 Dou Z, Ghosh K, Vizioli MG, Zhu J, Sen P, Wangensteen KJ, et al. Cytoplasmic chromatin triggers inflammation in senescence and cancer. Nature. 2017 Oct;550(7676):402-6.

26 Glück S, Guey B, Gulen MF, Wolter K, Kang TW, Schmacke NA, et al. Innate immune sensing of cytosolic chromatin fragments through cGAS promotes senescence. Nat Cell Biol. 2017 Sep;19(9):1061-70.

27 Patel S, Blaauboer SM, Tucker HR, Mansouri S, Ruiz-Moreno JS, Hamann L, et al. The common R71H-G230A-R293Q human TMEM173 is a null allele. J Immunol. 2017 Jan;198(2):776-87.
28 Goto M. Inflammaging (inflammation + aging): A driving force for human aging based on an evolutionarily antagonistic pleiotropy theory? Biosci Trends. 2008 Dec;2(6):218-30.

29 Bledowski P, Mossakowska M, Chudek J, Grodzicki T, Milewicz A, Szybalska A, et al. Medical, psychological and socioeconomic aspects of aging in Poland: assumptions and objectives of the PolSenior project. Exp Gerontol. 2011 Dec;46(12):1003-9.

30 Akbaraly TN, Hamer M, Ferrie JE, Lowe G, Batty GD, Hagger-Johnson G, et al. Chronic inflammation as a determinant of future aging phenotypes. CMAJ. 2013 Nov;185(16): E763-70.

31 Burdette DL, Monroe KM, Sotelo-Troha K, Iwig JS, Eckert B, Hyodo M, et al. STING is a direct innate immune sensor of cyclic diGMP. Nature. 2011 Sep;478(7370):515-8.

32 Hamann L, Bustami J, Iakoubov L, Szwed M, Mossakowska M, Schumann RR, et al. TLR-6 SNP P249S is associated with healthy aging in nonsmoking Eastern European Caucasians a cohort study. Immun Ageing. 2016 Mar;13: 7.

33 Thannickal VJ, Murthy M, Balch WE, Chandel NS, Meiners S, Eickelberg O, et al. Blue journal conference. Aging and susceptibility to lung disease. Am J Respir Crit Care Med. 2015 Feb; 191(3):261-9.

34 Murray MA, Chotirmall SH. The impact of immunosenescence on pulmonary disease. Mediators Inflamm. 2015;2015:692546.

35 Hewitt R, Farne H, Ritchie A, Luke E, Johnston SL, Mallia P. The role of viral infections in exacerbations of chronic obstructive pulmonary disease and asthma. Ther Adv Respir Dis. 2016 Apr;10(2):158-74.

36 Centers for Disease Control and Prevention (CDC). Cigarette smoking among adults United States, 2006. MMWR Morb Mortal Wkly Rep. 2007 Nov;56(44):1157-61.

37 Astuti Y, Wardhana A, Watkins J, Wulaningsih W; PILAR Research Network. Cigarette smoking and telomere length: A systematic review of 84 studies and meta-analysis. Environ Res. 2017 Oct;158:480-9.

38 Petrescu F, Voican SC, Silosi I. Tumor necrosis factor-alpha serum levels in healthy smokers and nonsmokers. Int J Chron Obstruct Pulmon Dis. 2010 Aug;5:217-22.

39 Giaimo S, d'Adda di Fagagna F. Is cellular senescence an example of antagonistic pleiotropy? Aging Cell. 2012 Jun;11(3):378-83.

40 López-Otín C, Blasco MA, Partridge L, Serrano M, Kroemer G. The hallmarks of aging. Cell. 2013 Jun;153(6):1194-217. 